\title{
Cerebellar Contributions to Locomotor Adaptations during Splitbelt Treadmill Walking
}

\author{
Susanne M. Morton ${ }^{1,2,3}$ and Amy J. Bastian ${ }^{3,4}$ \\ ${ }^{1}$ Departments of Physical Therapy and Rehabilitation Science and 2Anatomy and Neurobiology, University of Maryland School of Medicine, Baltimore, \\ Maryland 21201, and ${ }^{3}$ Department of Neurology and ${ }^{4}$ Kennedy Krieger Institute, Johns Hopkins University School of Medicine, Baltimore, Maryland 21205
}

Locomotor adaptability ranges from the simple and fast-acting to the complex and long-lasting and is a requirement for successful mobility in an unpredictable environment. Several neural structures, including the spinal cord, brainstem, cerebellum, and motor cortex, have been implicated in the control of various types of locomotor adaptation. However, it is not known which structures control which types of adaptation and the specific mechanisms by which the appropriate adjustments are made. Here, we used a splitbelt treadmill to test cerebellar contributions to two different forms of locomotor adaptation in humans. We found that cerebellar damage does not impair the ability to make reactive feedback-driven motor adaptations, but significantly disrupts predictive feedforward motor adaptations during splitbelt treadmill locomotion. Our results speak to two important aspects of locomotor control. First, we have demonstrated that different levels of locomotor adaptability are clearly dissociable. Second, the cerebellum seems to play an essential role in predictive but not reactive locomotor adjustments. We postulate that reactive adjustments may instead be predominantly controlled by lower neural centers, such as the spinal cord or brainstem.

Key words: cerebellum; motor learning; locomotion; central pattern generator; adaptation; human

\section{Introduction}

Locomotion is a mechanically complex task, yet animals walk using different patterns and through varied environments with astounding ease. In many animals, central pattern generators (CPGs) afford the spinal cord nearly autonomous control of basic locomotor rhythms [lamprey (Grillner, 1985; Grillner et al., 1995); frog (Arshavsky et al., 1993; Roberts et al., 1995); turtle (Lennard and Stein, 1977); cat (Brown, 1911; Grillner and Zangger, 1979; Rossignol et al., 1998) ]. In humans and other primates, the behavioral and neurophysiological evidence for CPGs remains incomplete, although studies of humans with spinal cord injury (Calancie et al., 1994; Dimitrijevic et al., 1998) and infant stepping patterns (Yang et al., 1998, 2004) support the existence of similar circuitry. Nonetheless, it is presumed that in human locomotion, the cerebellum and other supraspinal structures play a more critical role, because of the additional demands of bipedal walking (Grillner and Wallen, 1985; Armstrong, 1986).

The cerebellum has also been theorized to help provide adaptability to motor patterns. One hypothesis is that the cerebellum functions to process sensory inputs and make immediate alterations of ongoing movement patterns (Allen and Tsukahara, 1974; Shimansky et al., 2004). Thus, the cerebellum could act as a

Received March 28, 2006; revised July 29, 2006; accepted July 31, 2006.

This work was supported by National Institutes of Health Grant R01 HD040289. We thank P. S. Stein for thoughtful suggestions regarding study design and analyses, J. Choi and D. Reisman for their regular contributions to analyses and interpretation, and R. Bunoski and N. Zamora for their expertise with data collection.

Correspondence should be addressed to Dr. Susanne M. Morton, University of Maryland School of Medicine, Department of Physical Therapy and Rehabilitation Science, Allied Health Building, Suite 115, 100 Penn Street, Baltimore, MD 21201. E-mail: smorton@som.umaryland.edu.

DOI:10.1523/JNEUROSCI.2622-06.2006

Copyright $\odot 2006$ Society for Neuroscience $\quad$ 0270-6474/06/269107-10\$15.00/0 real-time sensory processing device (Bower, 1997), modulating motor responses in a reactive or feedback manner based on sensory perturbations. A different notion is that the cerebellum alters movement patterns in a predictive manner using trial-anderror practice (Thach et al., 1992). This hypothesis is consistent with several well known features of the cerebellum including its widespread capacity for plasticity in the cortex and nuclei (Ito, 1989, 2000; Hansel et al., 2001) and the behavioral evidence that cerebellar damage interferes with many forms of practicedependent motor adjustments (McCormick et al., 1985; Horak and Diener, 1994; Martin et al., 1996; Lang and Bastian, 1999). These predictive, or feedforward, adjustments require practice to obtain and result in storage of the new movement pattern (seen as negative aftereffects) after return to the original condition (Weiner et al., 1983; Shadmehr and Mussa-Ivaldi, 1994; Martin et al., 1996). The reactive or feedback-driven adaptations differ importantly from predictive adaptations in that they occur more quickly in response to ongoing afferent feedback (i.e., do not require practice) and are not stored by the nervous system (i.e., do not produce aftereffects).

We designed a locomotor task to test whether the cerebellum is required for reactive feedback motor adaptations versus predictive feedforward motor adaptations. The splitbelt treadmill provides a unique tool to study this question because healthy subjects demonstrate reactive adjustments of some walking parameters, such as stride length and time in stance, to accommodate the novel difference in treadmill belt speeds (Dietz et al., 1994; Reisman et al., 2005), but feedforward adaptation of other parameters, such as step length, time in double support, and interlimb phase relationships (Reisman et al., 2005). We could therefore test whether cerebellar damage in humans interferes 
Table 1. Cerebellar subject characteristics

\begin{tabular}{|c|c|c|c|c|c|c|c|c|}
\hline Subj & Age & Gend & Diagnosis & Time since onset & Belt speeds & ICARS total & Posture and gait & Limb kinetics \\
\hline CB-1 & 52 & $\mathrm{~F}$ & Idiopathic pancerebellar atrophy & 8.5 & $0.5,1.0$ & 30 & 11 & 10 \\
\hline CB-2 & 56 & $\mathrm{~F}$ & Hereditary pancerebellar atrophy & 11 & $0.4,0.8$ & 33 & 12 & 15 \\
\hline CB-3 & 31 & M & SCA 8 & 5 & $0.5,1.0$ & 35 & 12 & 15 \\
\hline CB-4 & 35 & $\mathrm{~F}$ & SCA 6 & 8 & $0.5,1.0$ & 40 & 11 & 23 \\
\hline CB-5 & 45 & M & Idiopathic pancerebellar atrophy & 25 & $0.5,1.0$ & 40 & 13 & 22 \\
\hline CB-6 & 48 & M & Idiopathic pancerebellar atrophy & 8 & $0.5,1.0$ & 41 & 14 & 19 \\
\hline CB-7 & 56 & M & Idiopathic pancerebellar atrophy & 5 & $0.4,0.8$ & 42 & 14 & 24 \\
\hline CB-8 & 45 & $\mathrm{~F}$ & Static pancerebellar atrophy & 33 & $0.5,1.0$ & 45 & 15 & 23 \\
\hline (B-9 & 49 & $\mathrm{~F}$ & Idiopathic pancerebellar atrophy & 14 & $0.4,0.8$ & 56 & 24 & 24 \\
\hline Mean: & $46.3 \pm 8.6$ years & & & $13.0 \pm 9.7$ years & & & & \\
\hline
\end{tabular}

Subj, Subject number; Gend, gender; F, female; M, male; SCA 6, spinocerebellar ataxia type 6; SCA 8, spinocerebellar ataxia type 8. Belt speeds are given in meters per second for the slow and fast conditions, respectively. Columns for posture and gait and limb kinetics refer to the two subscores of the ICARS. Means are given \pm 1 SD.

with the production of feedback and/or feedforward adjustments within the same task. Preliminary findings have been published previously in abstract form (Morton and Bastian, 2004).

\section{Materials and Methods}

Subjects. Seventeen individuals with cerebellar damage ( 7 females and 10 males) and 17 age- and gender-matched healthy control subjects were recruited to participate in the study. All subjects gave their informed consent before participating and a human studies committee approved the study. Cerebellar damage was confirmed by magnetic resonance imaging or computed tomography scan and all cerebellar subjects underwent a thorough motor neurological examination before testing. Subjects with radiological evidence of damage beyond the region of the cerebellum and/or clinical evidence of involvement of other brain structures (e.g., motor weakness, somatosensory loss, hyperreflexia, bradykinesia, rigidity) were excluded from the study. Part of the clinical examination included rating the severity of ataxia using the International Cooperative Ataxia Rating Scale [ICARS (Trouillas et al., 1997)]. The ICARS is an ordinal-scale clinical measure that rates ataxia in four movement categories: posture and gait, limb kinetics, speech, and oculomotor. To ensure that our sample of cerebellar subjects did in fact have significant gait ataxia, our inclusion criteria required a score of 30 or higher on the total ICARS and 10 or higher on the posture and gait ICARS subscore. Of the 17 subjects initially recruited, nine met these criteria. Data from these nine individuals [five females, four males; average age, $46.3 \pm 8.6$ (mean in years $\pm 1 \mathrm{SD}$ ); median total ICARS score, 40 (range, 30-56); median posture and gait ICARS subscore, 13 (range, 11-24); average time since onset, 13.0 years \pm 9.7] and their matched controls (five females, four males; average age, 46.3 years \pm 6.6 ) are reported here.

Table 1 provides information about the cerebellar group. All of the subjects had diffuse cerebellar damage caused by a degenerative disease, so we were unable to isolate the lesion to a particular cerebellar lobule, nucleus, or longitudinal zone. Thus, we could not directly test for the specificity of any results we might find to a particular region of the cerebellum. However, we did test whether the clinical presentation of limb ataxia versus gait ataxia affected adaptability by studying five additional cerebellar subjects. These individuals did not meet eligibility criteria and therefore were not included in the main study (total ICARS scores were $<30$ and/or posture and gait ICARS subscores were $<10$ ). Three of these individuals matched three subjects in the main cerebellar group with respect to their limb kinetics ICARS subscores (which reflects ataxia of voluntary isolated limb movements), but had much better posture and gait performance. The remaining two were similar to the three subjects in the main cerebellar group with regard to their posture and gait ICARS subscores, but had better voluntary limb movement control. This provided a way to compare performance between cerebellar subjects with different levels of limb or postural and gait ataxia. It is known that people with lateral cerebellar damage tend to have prominent limb ataxia with little impairment of balance and gait, whereas people with medial cerebellar damage have more profound deficits of balance and gait with fewer deficits of limb movements (Dichgans and Diener, 1985). We have used this type of behavioral categorization previously to infer involvement of different cerebellar regions in the control of walking (Morton and Bastian, 2003).

Paradigm. The paradigm consisted of walking on a custom-built treadmill (Woodway USA, Waukesha, WI) that has two belts, each with its own motor, so that the speed of each belt (i.e., each leg) can be controlled independently. During different testing periods, subjects walked on the treadmill with the two belts either moving at the same speed ("tied" configuration) or different speeds ("splitbelt" configuration). During the tied configuration, treadmill belt speeds were either "slow" $(0.5 \mathrm{~m} / \mathrm{s})$ or "fast" $(1.0 \mathrm{~m} / \mathrm{s})$. In the splitbelt configuration, one treadmill belt was set at the slow speed whereas the other was set at the fast speed. Three cerebellar subjects were unable to sustain walking at the $1.0 \mathrm{~m} / \mathrm{s}$ speed. For these subjects (and their matched healthy controls), treadmill belt speeds were reduced to $0.4 \mathrm{~m} / \mathrm{s}$ and $0.8 \mathrm{~m} / \mathrm{s}$ so that the splitbelt configuration always involved a $2-1$ ratio of fast to slow belt speeds.

Subjects participated in three testing periods. In the "baseline period," the belts were kept tied and moved first at the slow speed, then at the fast speed, and then again at the slow speed. In the "adaptation period," the treadmill was switched to the splitbelt configuration (one belt fast, the other belt slow). In the "postadaptation period," the belts were returned to the tied slow configuration. Figure 1 provides an illustration of the experimental paradigm. Before data collection, subjects walked on the treadmill (in the tied condition) briefly at various speeds, including the slow and fast speeds at which they would be subsequently tested. They were not given any practice in the splitbelt configuration, although they were told that the two belts would move at two different speeds at some point during the testing. For safety, all subjects held onto a front handrail and also wore a safety harness around the upper chest while walking on the treadmill. The harness was mounted to the ceiling and prevented falls but did not support body weight. The harness did not interfere with subjects' walking. Cerebellar subjects were tested with their more impaired leg on the faster moving belt during the splitbelt configuration. Matched control subjects were tested on the corresponding leg, accounting for leg preference. Leg preference was determined by which leg the subjects preferred to use to kick a ball.

An examiner controlled the treadmill from a remote computer and collected data continuously throughout all testing periods. The durations of each testing period were as follows: baseline, 3-4 min (tied slow), 8-10 min (tied fast), 4-5 min (tied slow); adaptation, $10 \mathrm{~min}$; and postadaptation, 4-5 min (Fig. 1a). Before the start of each period, subjects were told that the treadmill was about to start, but they were not told the speed or the belt coupling condition. Subjects were instructed to look straight ahead and refrain from looking down at the belts while walking so that they could not use visual information to determine belt speeds. An examiner stood by to monitor compliance with this instruction. After the first three or four strides of each period, subjects were asked whether they felt the two belts were moving at the same speed or two different speeds. If they thought the belts were moving at different speeds, they were asked to indicate which belt (right or left) was moving faster. At the end of each period, the treadmill was stopped and subjects were given a standing rest break of $\sim 2-3 \mathrm{~min}$. Treadmill acceleration and deceleration were always $0.3 \mathrm{~m} / \mathrm{s}^{2}$. 
A
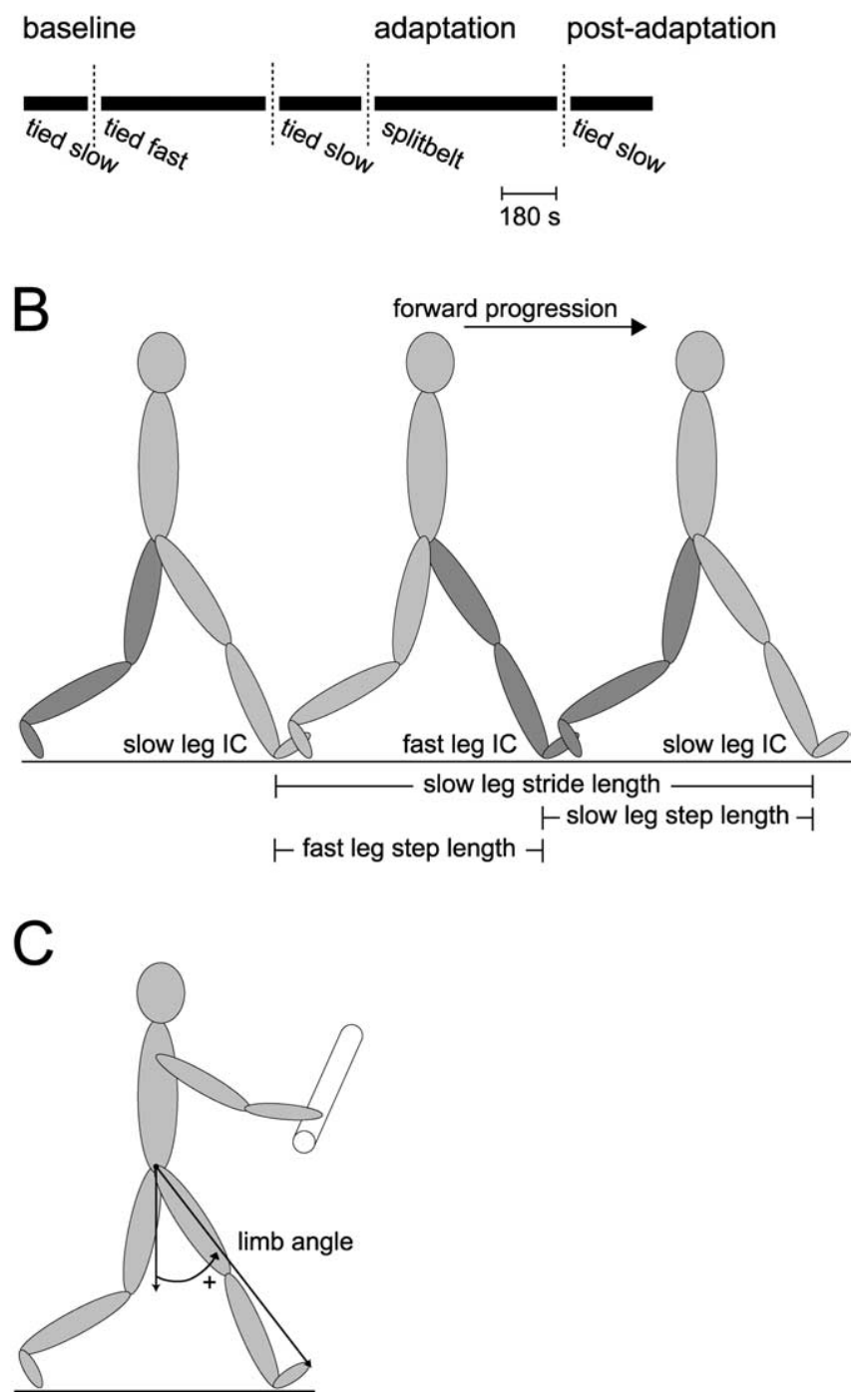

Figure 1. A, Time course for the experimental paradigm showing baseline, adaptation, and postadaptation periods and tied and splitbelt conditions. $\boldsymbol{B}$, Illustration of the method to calculate step and stride lengths. IC, Initial contact. The schematic depicts overground walking with a forward progression, but recall that during treadmill locomotion there is no real forward progression. Therefore, stride length could not be calculated as the distance the ankle traveled between subsequent initial contacts on a limb. Rather, we measured stride length as the distance the ankle traveled between initial contact and lift-off on a limb (for details, see Materials and Methods). C, Illustration of the method to calculate limb angles.

Data collection. Joint positions were recorded in three dimensions using the Optotrak System (Northern Digital, Waterloo, Ontario, Canada). Two Optotrak sensors were placed on opposite sides of the treadmill so that recordings could be made simultaneously from both sides of the body. Six infrared light-emitting diodes marked the positions of the hips (greater trochanter), ankles (lateral malleolus), and feet (head of fifth metatarsal). We defined the coordinates of our laboratory space such that anterior-posterior movements were in the $x$ direction, vertical movements in the $y$ direction, and medial-lateral movements in the $z$ direction. Position data were collected at $100 \mathrm{~Hz}$. Eight footswitches (Motion Lab Systems, Baton Rouge, LA) were placed on the toes and heels of subjects' shoes to record the times of initial contact and lift off of each foot from the ground. Footswitch and treadmill velocity data were collected at $1000 \mathrm{~Hz}$ and time-synchronized with the position data.
Data analysis. Joint position data were low-pass filtered at $8 \mathrm{~Hz}$. Optotrak software was used to calculate joint positions and angles. Custom software written in Matlab (The MathWorks, Natick, MA) was used for all subsequent analyses. Based on our previous work in healthy controls, we measured walking parameters that were expected to change quickly using reactive feedback-driven corrections and other parameters that were expected to change more gradually using predictive feedforward mechanisms (Reisman et al., 2005). The feedback parameters were stride length and the percentage time in stance phase. The feedforward parameters were step length and the percentage time in double limb support (Fig. $1 b$ ). These variables were specifically selected for analysis so we could compare analogous spatial (stride vs step lengths) and temporal (percentage times in stance vs double limb support) measures. We also calculated a third feedforward parameter, limb angle phasing, to quantify the phase relationship between the two limbs. The parameters were calculated as follows. Stride length was determined by the $x$ distance traveled by one of the ankle markers from the time of initial contact to the time of lift off on one limb. The percentage time in stance phase was calculated as the time from initial contact to lift off on one limb, expressed as a percentage of the total stride time (the time from initial contact to next initial contact) for that limb. Step length was calculated as the difference between the $x$ distances of the leading and trail limb ankle markers at the time of initial contact on the leading limb. The percentage time in double limb support was calculated as the time from initial contact on one limb to lift-off on the other limb, expressed as a percentage of the total stride time of the lift-off limb. All of these measures were calculated for both limbs. To calculate limb angle phasing, we first measured limb angle as the angle between a vector connecting the hip and metatarsal markers in the $x-y$ plane and a vertical line bisecting the hip marker (Fig. 1c). Phasing between the legs was calculated as the lag time (expressed as a percentage of stride time) at the peak of the cross-correlation function calculated between the two limb angles over the course of three consecutive stride cycles.

For each subject, walking parameters were averaged over the first and last five strides of each testing period to evaluate performance during the early and late portions, respectively, of each period. These values were then averaged for all subjects in each group. Thus, we compared walking parameters across late baseline, early and late adaptation, and early and late postadaptation periods. Statistical comparisons were completed using Matlab and Statistica (StatSoft, Tulsa, OK) software. For all walking parameters except limb angle cross-correlations, we used factorial ANOVA with repeated measures on one factor. The between-subjects factor was group (control and cerebellar) and the within-subjects (repeated measure) factor was testing period (late baseline, early and late adaptation, and early and late postadaptation). When the ANOVA yielded significant results, post hoc analyses were completed using Tukey's honest significant different test. For the limb angle phasing values, we calculated the mean vector and the angular deviation (the circular equivalents for mean and SD) and used the Watson's $U^{2}$ statistic to test for differences across testing periods (Batschelet, 1981). The level of statistical significance for all measures was set at $p<0.05$.

\section{Results}

For all results, we refer to the legs on the slower and faster moving belts during the splitbelt condition as the "slow" and "fast" legs, respectively (although during the baseline and postadaptation periods both legs are moving at the slow speed). Overall, performance by the control group was similar to that described previously (Reisman et al., 2005). Although all cerebellar subjects were able to maintain walking during each treadmill period, the group as a whole showed clearly contrasting levels of performance depending on the specific walking parameter assessed.

\section{Reactive feedback locomotor adaptations}

Figure 2 illustrates the walking parameters we expected to change quickly in a step-like function, stride length, and stance time. Stride length measures are shown on a stride-by-stride basis for a 
typical control subject and a cerebellar subject in Figure 2a. The control (top) and cerebellar subjects showed similar consistency and symmetry in stride lengths during the baseline period, although the cerebellar subject took shorter strides than the control during both the fast and slow speeds. During early adaptation, both subjects made rapid (within a few strides) reactive adjustments in stride lengths on the fast and slow legs, such that stride lengths increased substantially on the fast leg and decreased slightly on the slow leg. These alterations of stride lengths were maintained in both legs throughout adaptation. In postadaptation, both the control and cerebellar subjects again made quick feedback adaptations of stride length on the fast and slow legs, back to near-baseline levels. Figure $2 b$ shows stance times for two different control and cerebellar subjects. Results were similar, however, in that both subjects produced rapid reactive adaptations of the percentage time in stance during adaptation and postadaptation periods. During adaptation, the fast leg quickly decreased its stance time and the slow leg quickly (although slightly) increased its stance time; during postadaptation, the stance times of both legs rapidly returned to baseline levels. The cerebellar subject showed more stride-to-stride variability, but was still clearly able to generate the proper corrective response as quickly and to the same magnitude as the control subject.

Figure 2, $c$ and $d$, shows the group data, averaged over all subjects in the control (squares) and cerebellar (triangles) groups. Stride lengths are shown in Figure $2 c$, and stance times in Figure $2 d$. The statistical analyses revealed main effects of the testing period (all $p$ values $<0.001)$. Importantly, however, there were neither any main effects of group nor any group by testing period interaction effects (all $p$ values $>0.05$ ). So there were no differences between control and cerebellar groups for the reactive feedback adaptation of either of these walking parameters. For stride length (Fig. $2 c$ ), there was a significant increase on the fast leg (control group: from $0.42 \pm 0.07 \mathrm{~m}$, mean stride length $\pm 1 \mathrm{SD}$, to $0.61 \pm 0.13 \mathrm{~m}$; cerebellar group: from $0.44 \pm 0.06 \mathrm{~m}$ to $0.60 \pm 0.07 \mathrm{~m})$ and a significant decrease on the slow leg (control group: from $0.42 \pm$ $0.07 \mathrm{~m}$ to $0.38 \pm 0.09 \mathrm{~m}$; cerebellar group: from $0.44 \pm 0.05 \mathrm{~m}$ to $0.36 \pm 0.06 \mathrm{~m}$ ) that was maintained throughout adaptation (late baseline vs early adaptation, post hoc, all $p$ values $<0.01$ ). These adjustments were corrected immediately in postadaptation to near-baseline levels (late adaptation vs early postadaptation, post $h o c$, all $p$ values $<0.001$; early postadaptation vs late baseline, post $h o c$, all $p$ values $>0.05$ ). For stance times (Fig. $2 d$ ), the result was essentially the same. Stance time was increased on the slow leg (control group: from $66.4 \pm 3.1 \%$, mean stance time $\pm 1 \mathrm{SD}$, to $68.8 \pm 1.9 \%$; cerebellar group: from $67.6 \pm 3.2 \%$ to $69.2 \pm 4.6 \%$ ) and decreased on the fast leg (control group: from $67.0 \pm 2.9 \%$, mean stance time $\pm 1 \mathrm{SD}$, to $59.8 \pm 2.6 \%$; cerebellar group: from $67.3 \pm 1.9 \%$ to $60.5 \pm 1.3 \%$ ) within the first five strides of the

\section{stance time}
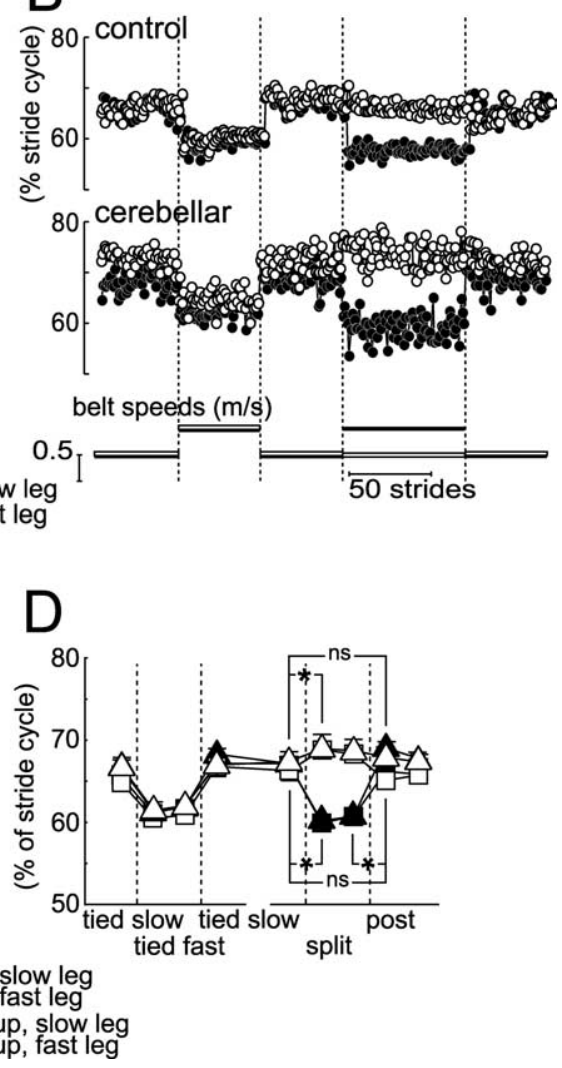

$\square$ control group, slow leg

$\triangle$ cerebellar group, slow leg

$\Delta$ cerebellar group, fast leg

Figure 2. Reactive feedback adaptations. $\boldsymbol{A}, \boldsymbol{B}$, Stride length $(\boldsymbol{A})$ and stance time $(\boldsymbol{B})$ values for the slow and fast legs for sequential strides on the treadmill from a typical control (top row) and cerebellar (bottom row) subject across all testing periods. fostrides are plotted for each component of the baseline period and for the postadaptation period; the first 75 strides are plotted for the adaptation period. $\boldsymbol{C}, \boldsymbol{D}$, Average stride length $(\boldsymbol{C}$ ) and stance time $(\boldsymbol{D})$ values on the slow and fast legs for control and indicate \pm 1 SE. Asterisks indicate a significance level of $p<0.05$ for the post hoc analysis; ns, not significant.

adaptation period (late baseline vs early adaptation, post hoc, all $p$ values $<0.01$ ) and an immediate correction of these changes back to baseline levels in early postadaptation (early postadaptation vs late baseline, post hoc, all $p$ values $>0.05$ ). Thus, during splitbelt treadmill locomotion, subjects with cerebellar damage were able to make rapid feedback-driven adaptations of their walking patterns that were appropriate and comparable with control subject performance.

\section{Predictive feedforward locomotor adaptations}

Figure 3 shows the walking parameters we expected to change more slowly over the course of adaptation, step length and double support time. Figure $3 a$ shows step-length values on a stride-bystride basis for the same control and cerebellar subjects shown in Figure $2 a$ (for stride length, the analogous spatial variable). Step lengths are plotted as the difference between the fast and slow legs (fast leg step length minus slow leg step length). In the baseline period, performance was characterized by step length differences at or near zero, indicating symmetry between the right and left legs regardless of treadmill speed. During adaptation, both subjects initially showed a great degree of asymmetry. Specifically, both took relatively longer steps on the slow leg and relatively shorter steps on the fast leg. However, the control subject gradually corrected the asymmetry so that by the end of the adaptation, the fast and slow legs moved similar distances once again. In contrast, the cerebellar subject showed only minimal improve- 


\section{step length}
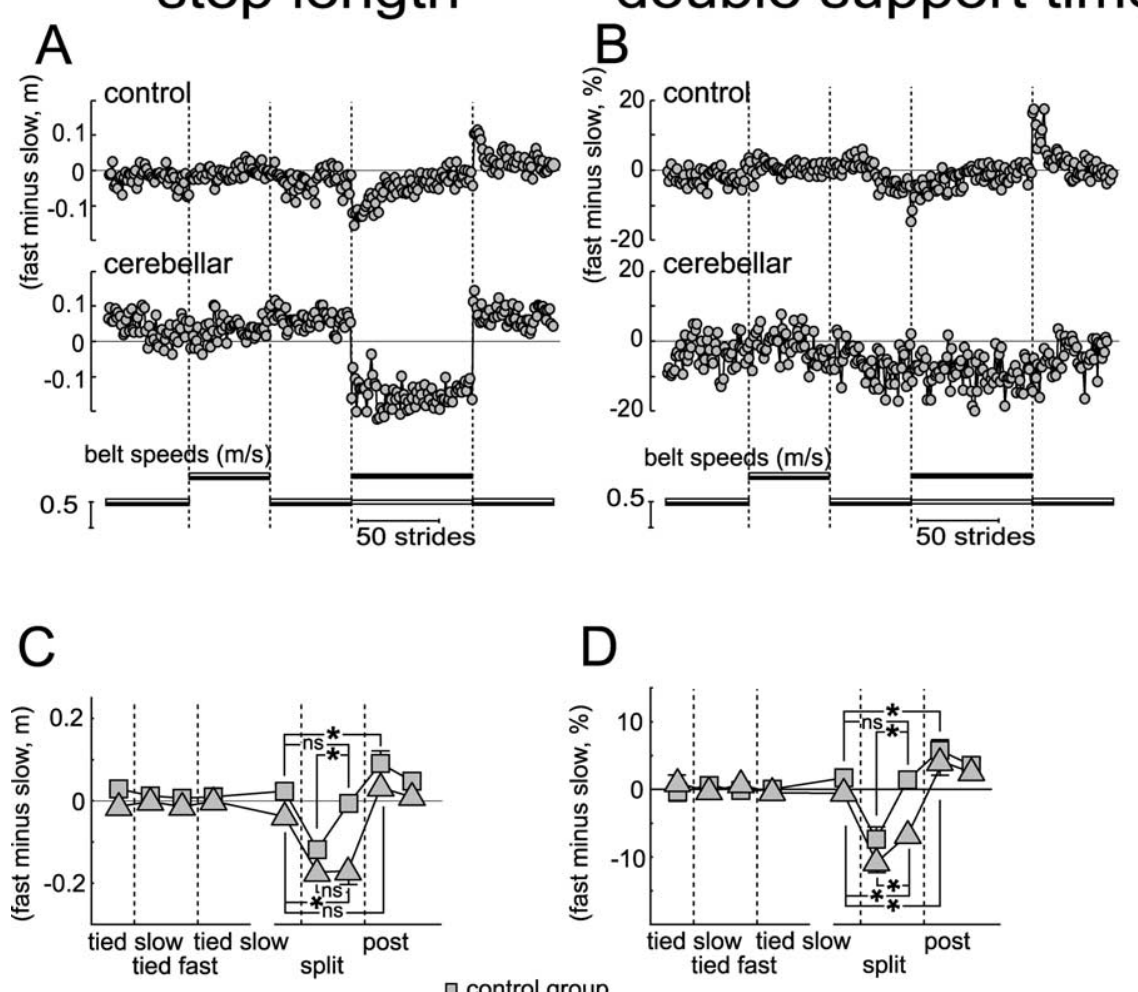

$\square$ control group

$\triangle$ cerebellar group

\section{double support time}

D

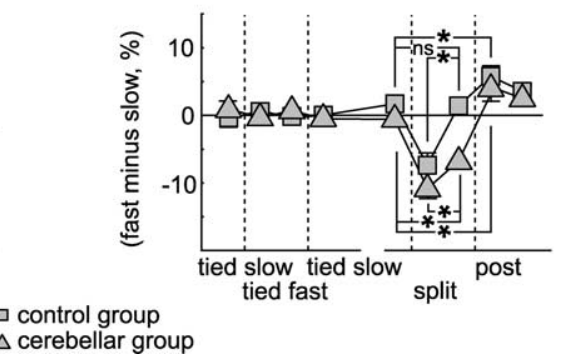

Figure 3. Predictive feedforward adaptations. $\boldsymbol{A}, \boldsymbol{B}$, Step length $(\boldsymbol{A})$ and double support time $(\boldsymbol{B})$ values for sequential strides on the treadmill from a typical control (top row) and cerebellar (bottom row) subject across all testing periods. The control and cerebellar subjects in $\boldsymbol{A}$ are the same subjects as shown in Figure $2 A$; the control and cerebellar subjects in $\boldsymbol{B}$ are the same subjects as shown in Figure $2 B$. The first 50 strides are plotted for each component of the baseline period and for the postadaptation period; the first 75 strides are plotted for the adaptation period. Circles indicate the difference between the legs (fast leg minus slow leg) in step length and double support time values. $\boldsymbol{C}, \boldsymbol{D}$, Average step length $(\boldsymbol{C})$ and double support time $(\boldsymbol{D})$ differences for control and cerebellar groups. Each data point represents values averaged over the early or late portions of each testing period. Error bars indicate $\pm 1 \mathrm{SE}$. Asterisks indicate a significance level of $p<0.05$ for the post hoc analysis; ns, not significant.

tation and also failed to show a negative aftereffect in early postadaptation.

Group data for these walking parameters are provided in Figure 3, $c$ and $d$. Step length differences are shown in Figure $3 c$ and double support time differences in Figure $3 d$. For both parameters, the statistical analysis revealed significant group by testing period interaction effects (all $p$ values $<0.05)$, indicating that control and cerebellar groups showed different levels of performance over the different testing periods. For step length (Fig. 3c), the initial asymmetries during early adaptation were of a similar magnitude in both groups (control: $-0.11 \pm 0.04 \mathrm{~m}$, mean difference in step lengths $\pm 1 \mathrm{SD}$; cerebellar: $-0.17 \pm 0.06 \mathrm{~m}$, post hoc, $p>0.05)$. Yet only the control group was able to correct the asymmetry over the course of adaptation (late adaptation, mean $-0.01 \pm$ $0.05 \mathrm{~m}$, early vs late adaptation, post hoc, $p<0.01$; late baseline vs late adaptation, post hoc, $p>0.05)$. The cerebellar group showed no significant improvement in step-length differences over the course of adaptation and therefore did not approach baseline levels by the end of adaptation (late adaptation, mean $-0.17 \pm$ $0.11 \mathrm{~m}$; early vs late adaptation, post hoc, $p>0.05$; late baseline vs late adaptation, posthoc, $p<0.001)$. In postadaptation, the control group showed a significant negative aftereffect in step length differences (early postadaptation, mean $0.09 \pm 0.08$ $\mathrm{m}$; late baseline vs early postadaptation, post hoc, $p<0.05$ ), which is indicative of storage of a predictive motor adaptation

ment of the asymmetry over the course of adaptation. With the return to tied belts in postadaptation, the control initially showed the reverse asymmetry (negative aftereffect). That is, the subject took a relatively shorter step on the slow leg and a relatively longer step on the fast leg. After several strides, the subject gradually corrected step lengths on both legs and returned to a symmetric step pattern. The cerebellar subject, however, showed only a small negative aftereffect that did not return to symmetry. Figure $3 b$ shows double support times on a stride-by-stride basis for the same control and cerebellar subjects shown in Figure $2 b$ (for stance time, the analogous temporal variable). The plots show differences in double support times: fast leg double support minus slow leg double support. As with step lengths, both the control and cerebellar subject had double support time differences near zero during the baseline period, indicating the expected symmetry. In early adaptation, both subjects initially showed a substantial asymmetry; specifically, a relatively longer double support time on the slow leg and a relatively faster double support time on the fast leg. The control subject gradually corrected the asymmetry so that by the end of adaptation the fast and slow legs spent nearly equal amounts of time in double support, and then in postadaptation demonstrated the expected reverse asymmetry (negative aftereffect), which was also gradually corrected. In contrast, the cerebellar subject did not show the gradual improvement in double support time symmetry over the course of adap-
(Weiner et al., 1983; Shadmehr and Mussa-Ivaldi, 1994). In contrast, the cerebellar group did not show a negative aftereffect in step-length differences (early postadaptation, mean $0.04 \pm 0.05$ $\mathrm{m}$; late baseline vs early postadaptation, post hoc, $p>0.05)$. The results were very similar for the double support time measures (Fig. $3 d$ ). Both groups showed similar initial asymmetries during early adaptation (control: $-7.4 \pm 5.6 \%$, mean difference in double support times $\pm 1 \mathrm{SD}$; cerebellar: $-10.6 \pm 5.0 \%$, post hoc, $p>$ $0.05)$. The control group corrected the asymmetry over the course of adaptation (late adaptation, mean $1.3 \pm 3.1 \%$; early vs late adaptation, post hoc, $p<0.001$; late baseline vs late adaptation, post hoc, $p>0.05$ ) and subsequently demonstrated a significant negative aftereffect in early postadaptation (early postadaptation, mean $5.8 \pm 4.7 \%$; late baseline vs early postadaptation, post hoc, $p<0.05$ ). In contrast, the cerebellar group showed only a moderate improvement in the double support asymmetry during adaptation and did not ever reach near-baseline (late adaptation, mean $-6.4 \pm 4.4 \%$; early vs late adaptation, post hoc, $p<$ 0.05 ; late baseline vs late adaptation, post hoc, $p<0.01$ ). In postadaptation, the cerebellar group showed a reduced (although significant) negative aftereffect (early postadaptation, mean $4.0 \pm$ $5.9 \%$; late baseline vs early postadaptation, post hoc, $p<0.05$ ). In summary, the cerebellar group showed a reduction (double support) or complete inability (step length) to adjust both types of predictive feedforward adapting walking parameters. The ac- 


\section{limb angle}

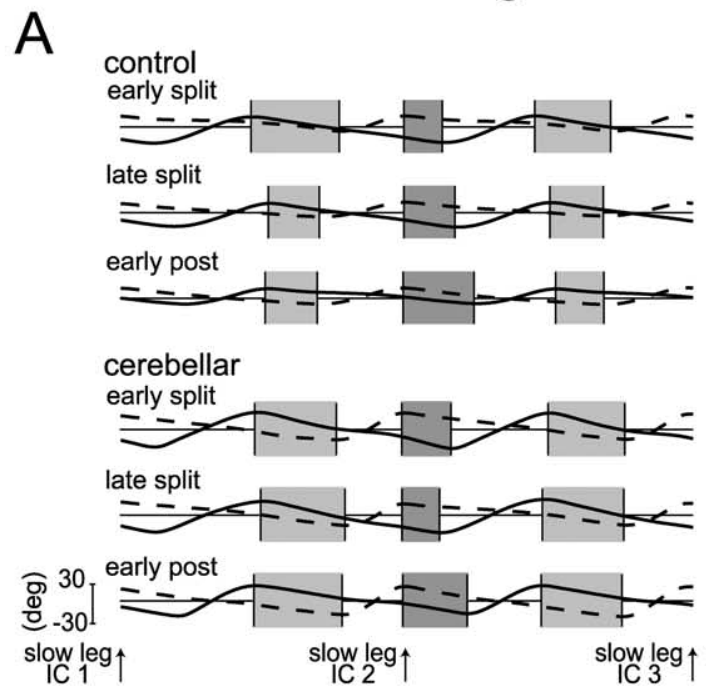

\section{limb angle interlimb phasing}
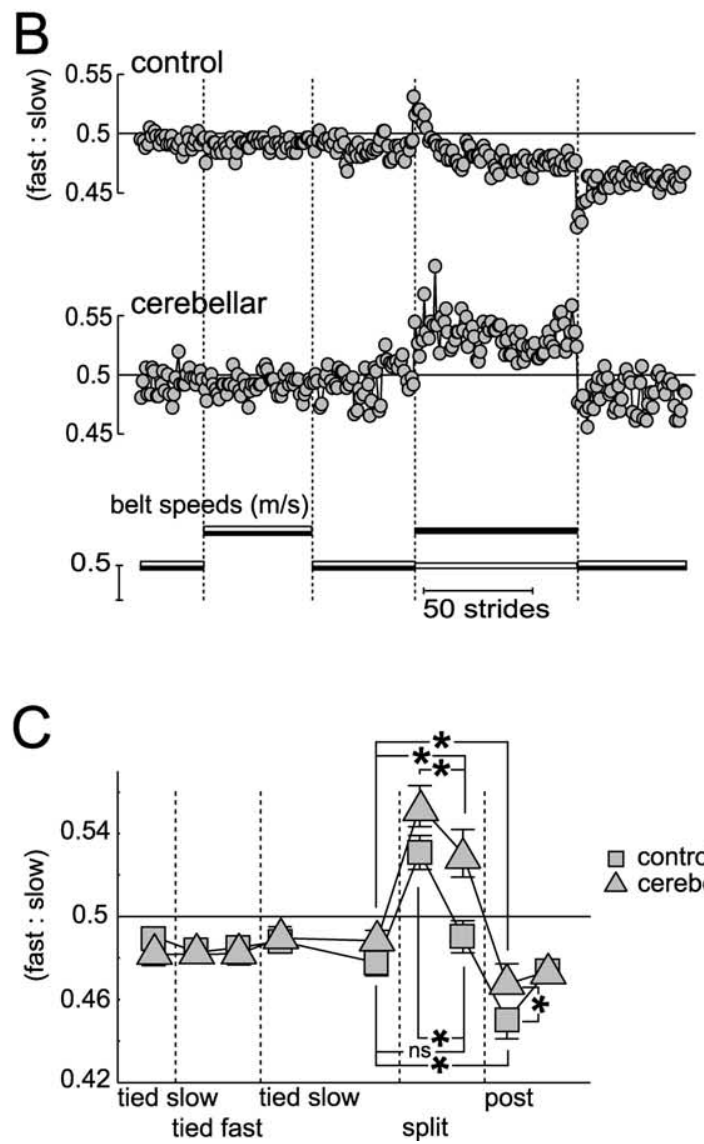

$\square$ control group $\triangle$ cerebellar group

Figure 4. Limb angle interlimb phase. $\boldsymbol{A}$, Limb angles on the slow (dashed line) and fast (solid line) legs plotted over two successive strides from a typical control (top three pairs of traces) and cerebellar (bottom three pairs of traces) subject. Pairs of strides are from the early adaptation period (top), the late adaptation period (middle), and the early postadaptation period (bottom). Stride times are normalized to percentage of stride for ease of viewing. All strides are aligned on the first initial contact (IC) on the slow leg (arrows indicate the times of slow leg contact). Light gray bars show the duration from peak limb flexion on the fast leg to peak limb extension on the slow leg; dark gray bars show the duration from peak limb flexion on the slow leg to peak limb extension on the fast leg. During symmetric walking, these two durations are equal; we show them here to illustrate the clear temporal shift in limb angles that

companying supplementary videos (supplemental movies 1,2 , available at www.jneurosci.org as supplemental material) compare the changes in these practice-dependent motor behaviors across the different testing periods for a control and a cerebellar subject.

In addition to these motor aftereffects, all control subjects always misperceived the belt speeds in the postadaptation period, judging the leg that had been on the slow belt during adaptation to be moving faster during early postadaptation, although the belts were moving at the same speed. Only two of nine cerebellar subjects showed this perceptual aftereffect. This appeared to be related to their deficient motor adaptation and not a general perceptual deficit because all subjects could correctly detect the speed difference between the two treadmill belts during the early splitbelt (i.e., adaptation) period of the experiment.

\section{Interlimb phasing}

Previously, we showed in healthy controls that a simple phase shift between limb motions is enough to produce the asymmetries of step length and double support times observed during early adaptation. The leg on the fast belt is initially pulled backward faster than expected, introducing a phase advancement of the fast leg position relative to the slow leg (Reisman et al., 2005). Correction of the phase shift back to an approximate 0.5 interlimb coordination pattern (i.e., legs $180^{\circ}$ out of phase with one another) returns the step length and double support times back to symmetric patterns. We therefore wanted to examine the phase relationship between the legs in these subjects. Figure 4 illustrates the interlimb phase relationship of limb kinematics. Limb angles on the fast and slow legs are shown in Figure $4 a$ during the early adaptation, late adaptation, and early postadaptation periods for a control and a cerebellar subject. Fast (solid) and slow (dashed) leg limb angles are overlaid and plotted for two successive strides in each period. The light gray bars denote the times from peak limb flexion on the fast leg to peak limb extension on the slow leg; dark gray bars indicate the times from peak limb flexion on the slow leg to peak limb extension on the fast leg. When the interlimb phase is 0.5 , the widths of all the bars are equal. In early adaptation, the splitbelt configuration produced a phase shift whereby the fast leg was phase advanced relative to the slow leg. This lengthened the time from fast leg peak flexion to slow leg peak extension (light gray bars) and shortened the time from slow leg peak flexion to fast leg peak extension (dark gray bars), as seen in the uppermost traces for the control and cerebellar subjects. By late adaptation, the control subject was able to adapt interlimb parameters and return the interlimb phasing to nearly 0.5 (as seen by the relatively equal widths of all the bars), but the cerebellar subject was not (light gray bars remain wider than darker bars). Similarly, the control subject appeared to store

occurs during the early adaptation and early postadaptation periods. These phase shifts are quantified over the duration of the limb angle cycle in the cross-correlation measures. $\boldsymbol{B}$, Limb angle interlimb phasing values for sequential strides on the treadmill from the same control (top) and cerebellar (bottom) subject shown in $\boldsymbol{A}$. The first 50 strides are plotted for each component of the baseline period and for the postadaptation period; the first 75 strides are plotted for the adaptation period. Circles indicate the lag time at the peak in the crosscorrelation function between the two limb angles; a lag time of 0.50 indicates the ideal interlimb phase relationship of $180^{\circ}$ out of phase. C, Average limb angle interlimb phasing values for the control and cerebellar groups. Each data point represents the lag time at the peak in the cross-correlation function between the two limb angles, averaged over the early or late portions of each testing period. Error bars indicate $\pm 1 \mathrm{SE}$. Asterisks indicate a significance level of $p<$ 0.05 for the post hoc analysis; ns, not significant. 
the adaptation, demonstrating a negative aftereffect in the early postadaptation period. The cerebellar subject did not show a negative aftereffect, but instead persisted in the asymmetric pattern induced by the splitbelt treadmill well into early postadaptation.

We quantified the interlimb phase relationship using crosscorrelation functions. Figure $4 b$ depicts the lag time at the peak in the cross-correlation function calculated between the two limb angles (fast to slow) for the same control and cerebellar subjects shown in Figure $4 a$. Throughout the baseline period, the limb angle interlimb phasing values for both subjects were near 0.5 , indicating that the two legs remained $180^{\circ}$ out of phase with one another at all times, regardless of treadmill speed. In early adaptation, both subjects initially showed a phase shift of $\sim 10 \%$ (from 0.50 to $\sim 0.55$ ) as described above, reflecting the phase advancement of the fast leg. The control subject gradually corrected the phase shift over the course of adaptation and showed a negative aftereffect in postadaptation, which was also gradually corrected. However, the cerebellar subject did not show any correction of the phase advancement over the course of adaptation and also showed only a minimal negative aftereffect after return to the tied belt configuration in early postadaptation.

Group results for the limb angle interlimb phasing values are provided in Figure $4 c$. The statistical analyses revealed a significant group by testing period interaction effect $(p<0.05)$. In early adaptation, both groups showed the expected phase shift in limb angles (control: $0.53 \pm 0.02$, mean lag times $\pm 1 \mathrm{SD}$; cerebellar: $0.55 \pm 0.03$, post hoc, $p>0.05$ ), yet only the control group was able to correct the phase shift over the course of adaptation (late adaptation, mean $0.49 \pm 0.02$; early vs late adaptation, post hoc, $p<0.001$; late baseline vs late adaptation, post hoc, $p>0.05$ ). Controls also demonstrated a significant negative aftereffect (early postadaptation, mean $0.45 \pm 0.03$; late baseline vs early postadaptation, $p<0.05$ ). The cerebellar group did not return to near-baseline levels by the end of adaptation (late adaptation, mean $0.53 \pm 0.03$, late baseline vs late adaptation, post hoc, $p<$ 0.05 ), and although they were able to produce a small negative aftereffect (early postadaptation, mean $0.46 \pm 0.03$; late baseline vs early postadaptation, $p<0.05$ ), it was significantly smaller than the controls (control vs cerebellar, post hoc, $p<0.05$ ).

\section{Relationship between forms of ataxia and predictive adaptive capabilities}

Figure $5 a$ shows, for the cerebellar group, that posture and gait ICARS subscores negatively correlated with the magnitude of the aftereffects of the predictive adaptation $(r=-0.58 ; p<0.05)$. In other words, subjects with lower (more normal) gait ataxia ratings had higher (better) aftereffects in the step-length difference measure. In contrast, limb kinetics ICARS subscores did not predict step-length aftereffects $(r=-0.42 ; p>0.05)$. We also compared performance between three subgroups of cerebellar subjects. Subgroup 1 consisted of three subjects who were participants in the main experiment (cerebellar subjects CB-1, -2, and -3). Subgroups 2 and 3 consisted of five subjects (two in subgroup 2 and three in subgroup 3 ) who were not eligible for the main experiment but who had uniquely disparate levels of gait versus limb ataxia. Subjects in subgroup 2 had deficits of posture and gait (i.e., posture and gait ICARS subscores were relatively high) with minimal deficits of voluntary limb movements (i.e., limb kinetics ICARS subscores were relatively low). Subjects in subgroup 3 had deficits of voluntary limb movements with minimal deficits of posture and gait (Fig. 5b). Subgroup 3, but not subgroups 1 or 2, adapted step lengths fully, as shown by their decreased residual error at the end of the adaptation period (Fig.
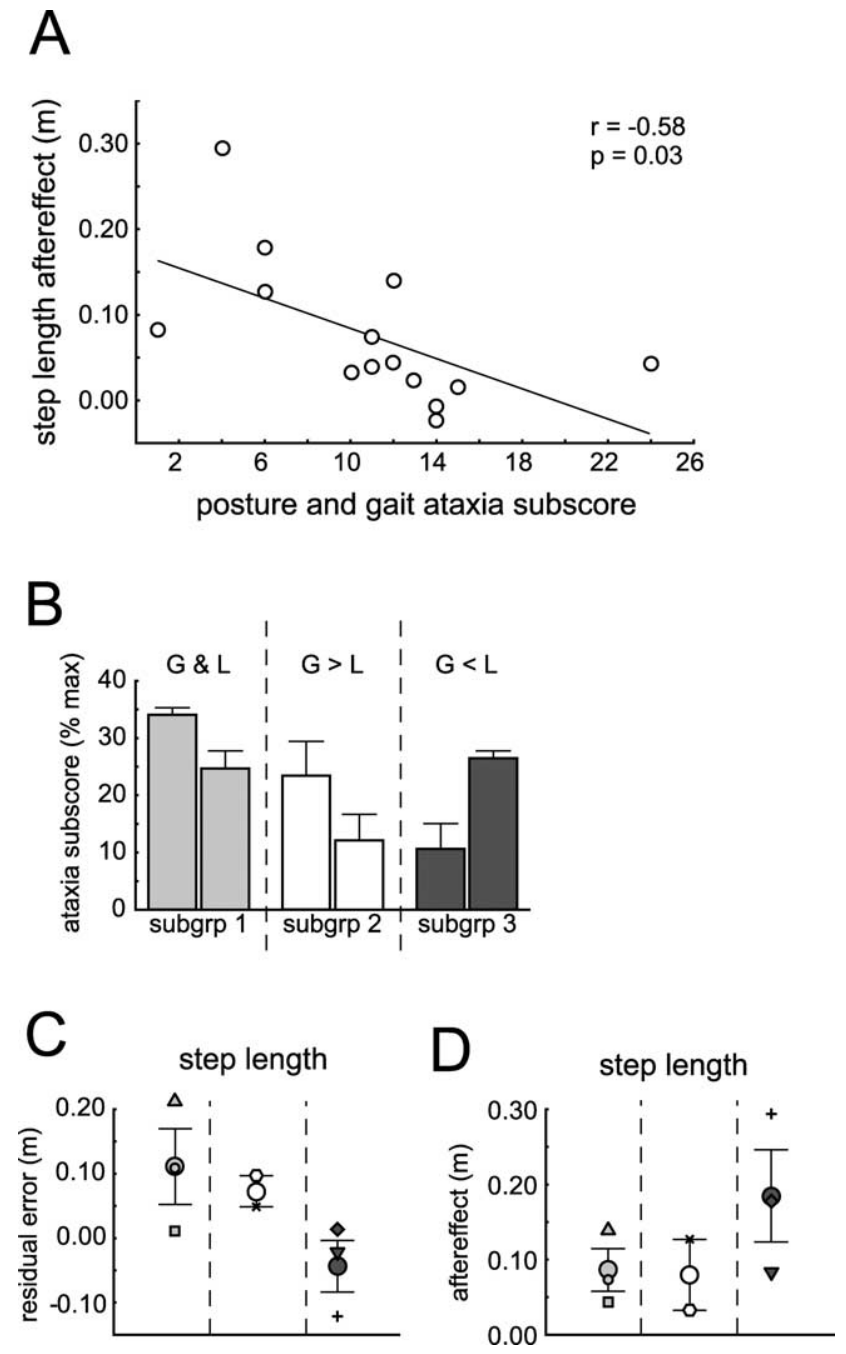

Figure 5. $\boldsymbol{A}$, Scatter plot showing the relationship between the posture and gait ICARS subscore and the capability for predictive adaptability (as measured by the magnitude of the negative aftereffect in the step length difference measure) for all subjects in the cerebellar group plus the five additional cerebellar subjects recruited for this analysis. The diagonal line indicates the fit derived from the correlation equation. $\boldsymbol{B}$, Posture and gait (left bar in each of the three pairs) and limb kinetics (right bar) ICARS subscores for three subgroups of cerebellar subjects. Subscore values are expressed in percentages, or the raw score for each category divided by the maximum possible score for that category, multiplied by 100 . Light gray bars indicate average data from cerebellar subgroup 1 (subjects (B-1, -2 , and -3), who had evidence of ataxia of both gait and posture and voluntary limb movements. Open bars depict average data from cerebellar subgroup 2, two new cerebellar subjects who were not part of the main study because their total ICARS scores were not high (severe) enough to meet the inclusion criteria. These subjects had clinical evidence of gait ataxia with only minimal evidence of limb ataxia. Dark gray bars show average data from cerebellar subgroup 3, three other new cerebellar subjects who were also not part of the main study because they did not meet inclusion criteria for total ICARS scores. They had clinical evidence of limb ataxia with only minimal gait and posture deficits. $C, D$, Average predictive adaptation performance for the same three subgroups of cerebellar subjects. Large circles represent average performance by each subgroup (light gray, subgroup 1; open, subgroup 2; dark gray, subgroup 3). Individual subjects are indicated with the smaller symbols: triangle, $C B-3$; circle, $C B-1$; square, $C B-2$; hexagon, a 62year-old male with a total ICARS score of $12 ; x$, a 70 -year-old male with a total ICARS score of 22; diamond, a 20-year-old male with a total ICARS score of 24; inverted triangle, a 40-year-old female with a total ICARS score of 17; cross, a 30-year-old female with a total ICARS score of 23. C, Average step length residual errors for the cerebellar subgroups. Residual errors were calculated as the difference in step-length difference values from the late baseline to the late adaptation periods. A score of zero indicates full predictive adaptability (a return to baseline levels); positive values indicate a failure to return to baseline, or poor predictive adaptability; negative values indicate late adaptation values that exceeded baseline levels. $\boldsymbol{D}$, Average step length negative aftereffects for the cerebellar subgroups. Negative aftereffects were taken from the early postadaptation period. Error bars indicate $\pm 1 \mathrm{SE}$. 
5c). Similarly, subgroup 3 also showed greater aftereffects than subgroups 1 or 2 (Fig. $5 d$ ). Although the number of subjects in each subgroup was small, the results suggest that cerebellar deficits of posture and gait, but not limb ataxia, are associated with poorer predictive adaptability during splitbelt treadmill locomotion.

\section{Discussion}

We demonstrated that cerebellar damage does not impair the ability to make reactive feedback-driven adaptations, but significantly disrupts predictive feedforward motor adaptations during splitbelt treadmill locomotion. Our results reveal an important distinction between different components of adaptability and their neural bases. Namely, predictive practice-dependent motor adaptations of human locomotion appear to be dependent on cerebellar integrity, whereas reactive adaptations during the same locomotor task are not. Recently, Smith et al. (Smith et al., 2000; Smith and Shadmehr, 2005) showed that during reaching in a novel force-field environment, cerebellar damage impairs the ability to make predictive corrective movements using information gained from previous trials, but does not interfere with the ability to make feedback-driven corrections during ongoing movements. Similar results have also been reported for grasping, with cerebellar subjects showing impairments in predictive finger forces to resist a self-generated perturbation, but more normal reactive finger forces to an external perturbation (Nowak et al., 2004). These findings and ours are similar and, together, suggest that (1) the cerebellum plays the same fundamental role in adjusting voluntary arm and hand movements as it does in adjusting less consciously controlled locomotor movements, and (2) that role involves using error feedback information to update motor commands for the generation of subsequent movements.

One caveat to remember with lesion studies is that they only provide information about what the nervous system cannot do without a given structure, and do not directly point to the role of the structure in the context of a normal brain. If there is a clear deficit in behavior, it suggests that the lesioned structure is required for that behavior. This seems to be the case for our predictive adaptation. However, if there is no deficit in behavior, it is not clear whether another part of the brain has compensated for the lesion, or whether that structure was unimportant for the behavior to begin with. We can conclude that the cerebellum is not needed for reactive locomotor adaptations, but we cannot say whether or not, in the intact state, the cerebellum is involved in these sorts of adaptations. Electrophysiological studies may better determine the role of the cerebellum in these types of locomotor adaptations.

\section{Levels of locomotor adaptability}

Successful locomotion in the environment requires the capability to make relatively fast-acting adjustments, such as a quick postural response to an unexpected movement of the support surface (e.g., stepping onto loose gravel), as well as more gradually emerging adjustments that require a period of exposure to or practice in the novel condition. The current findings support the idea that these different levels of locomotor adaptability might have separate neural substrates. Other recent studies have also focused on identification of these different types of locomotor adaptation. One recent study reported on human walking patterns when one leg was placed in a robotic gait orthosis that applied a viscous resistance to the leg (Lam et al., 2006). Subjects showed two adaptation patterns: first, a feedback adaptation where muscle activity was immediately increased during swing phase and immediately reduced again during catch (i.e., no resistance applied) trials, and second, a feedforward adaptation where muscle activation changes occurred before the perturbation (during the preswing period), appeared only after a bout of practice wearing the orthosis, and persisted during the catch trials (Lam et al., 2006). Pearson (2000) has also described these different levels as short- and long-term adaptations, respectively.

\section{Reactive feedback adaptations do not require cerebellar control}

The present findings seem to indicate that feedback-driven locomotor adaptations are not dependent on the cerebellum. The rapid reactive adjustments of stride length and stance and swing times were not impaired in subjects who had significant cerebellar damage. This is in agreement with another human locomotor study where subjects with cerebellar damage rapidly changed locomotor patterns to walk up an incline (Earhart and Bastian, 2001). Spinal structures may play an important role in these sorts of reactive locomotor adaptations. In cats and lower vertebrates, spinal CPGs are sufficient to produce the basic quadruped locomotor pattern (Grillner and Zangger, 1979; Rossignol et al., 1999) and, by definition, control movement without supraspinal influences or afferent feedback. When sensory information is available, spinal structures alone also provide some degree of flexibility to the basic locomotor pattern (Forssberg et al., 1975, Pearson, 1995). For example, position and load information from the ankle and especially the hip joint strongly influences the timing of the stance-to-swing transition (Grillner and Rossignol, 1978; Duysens and Pearson, 1980).

Splitbelt treadmill locomotion has been tested in decerebrate (Kulagin and Shik, 1970) and in spinalized (Forssberg et al., 1980) cats. One hindlimb is made to go faster than the others, and the animals immediately compensate by prolonging stance and shortening swing on the "slow" hindlimb, and vice versa on the "fast" hindlimb, just as we see in bipedal walking (Kulagin and Shik, 1970; Forssberg et al., 1980). This suggests that spinal circuits are capable of using sensorimotor information from one limb to alter the motor output of all limbs. Similar findings have been reported during supported stepping in human infants, whose descending pathways are still mostly unmyelinated (Thelen et al., 1987; Pang and Yang, 2000; Yang et al., 2004). Thus, both quadrupeds and human infants can make reactive feedback adaptations of stance and swing times to maintain a reciprocal leg movement pattern during splitbelt treadmill locomotion, relying predominantly (infant work) if not exclusively (cat work) on spinal circuitry.

Predictive feedforward adaptations require cerebellar control We have shown that step length and double support time during splitbelt walking are adapted with practice and the changes persist beyond the adaptation period. This adaptation is predictive in nature, depending on previous experience for its formation. Our subjects with cerebellar damage had reduced and sometimes absent adaptation of these walking parameters. Thus, the cerebellum seems to be more critical for this type of step-by-step adaptation. Decerebrate cats have been reported to maintain the capability for this type of locomotor adaptability (Yanagihara et al., 1993), indicating that cerebral cortical structures are not essential for the adaptation. Rather, evidence points to the role of the cerebellum in the splitbelt adaptive process. For example, Purkinje cell complex spike rates in the cerebellum increase dramatically during the initial period of exposure to the splitbelt treadmill (Yanagihara and Udo, 1994). And nitric oxide depriva- 
tion, which is thought to play a role in long-term depression, abolishes the adaptive behavior altogether in these cats (Yanagihara and Kondo, 1996). Thus, it appears that the cerebellum is critical for the adaptation of double support and step length patterns during splitbelt treadmill locomotion in quadrupeds and in humans. These specific walking parameters may be driven to adapt because their symmetry is critical for a smooth and stable locomotor pattern (Matsukawa et al., 1982; Yanagihara et al., 1993).

The current study cannot provide direct evidence for which cerebellar regions are most critical for the feedforward predictive adaptations. However, the comparison between cerebellar subjects with different levels of limb versus gait ataxia suggests that cerebellar regions controlling posture and gait may play a more important role in the control of feedforward gait adaptations than regions controlling voluntary limb movements. Other evidence from studies of humans (Dichgans and Diener, 1985) and monkeys (Sprague and Chambers, 1953; Chambers and Sprague, 1955a,b; Thach et al., 1992) indicates that the cerebellar regions most important for control of posture and locomotion are the midline vermis and fastigial nuclei.

\section{Cerebellar locomotor adaptations}

The current findings support the hypothesis that the cerebellum helps predict appropriate limb movements based on a stored internal representation (Maschke et al., 2004; Smith and Shadmehr, 2005) of, in this case, the desired interlimb relationship. Error information from sensorimotor feedback is presumed to drive alterations of and continuously update this representation (Ito, 1989, 2000; Kawato and Gomi, 1992; Imamizu et al., 2000). In the cat, mossy fiber projections from dorsal or ventral spinocerebellar tracts provide the cerebellum with the necessary feedback in several forms, including proprioceptive primary afferents (Lundberg and Oscarsson, 1956; Lundberg and Winsbury, 1960; Eccles et al., 1961) and information integrated across multiple joints of the leg, which may formulate an estimate of the foot endpoint location (Bosco and Poppele, 1997; Bosco et al., 2000). Some feedback is also integrated across both hindlimbs to provide a single cerebellar hemisphere with bilateral limb orientation information, which could be critical for adapting interlimb coordination (Poppele et al., 2003). Several studies have reported that locomotor perturbations cause climbing fiber discharge rates to increase (Andersson and Armstrong, 1987; Lou and Bloedel, 1992; Yanagihara and Udo, 1994), suggesting that these fibers may provide some sort of error or event signal to the cerebellum (Ito, 1989, 2000; Kawato and Gomi, 1992; Imamizu et al., 2000). During splitbelt locomotion, climbing fibers may signal the occurrence of a destabilizing event, for example, the leg on the faster belt being propelled backward sooner than anticipated, which could be perceived by the nervous system as a slip. Through the adaptive process, however, adjustments in the spatiotemporal relationship between the legs reduce the instability and the error, and critical components of interlimb symmetry are restored.

\section{References}

Allen GL, Tsukahara N (1974) Cerebrocerebellar communication systems. Physiol Rev 54:957-1006.

Andersson G, Armstrong DM (1987) Complex spikes in Purkinje cells in the lateral vermis (b Zone) of the cat cerebellum during locomotion. J Physiol (Lond) 385:107-1 34 .

Armstrong DM (1986) Supraspinal contributions to the initiation and control of locomotion in the cat. Prog Neurobiol 26:273-361.

Arshavsky YI, Orlovsky GN, Panchin YV, Roberts A, Soffe SR (1993) Neuronal control of swimming locomotion: analysis of the pteropod mollusc
Clione and embryos of the amphibian Xenopus. Trends Neurosci $16: 227-233$.

Batschelet E (1981) Circular statistics in biology. New York: Academic.

Bosco G, Poppele RE (1997) Representation of multiple kinematic parameters of the cat hindlimb in spinocerebellar activity. J Neurophysiol 78:1421-1432.

Bosco G, Poppele RE, Eian J (2000) Reference frames for spinal proprioception: limb endpoint based or joint-level based? J Neurophysiol 83:2931-2945.

Bower JM (1997) Control of sensory data acquisition. Int Rev Neurobiol 41:489-513.

Brown TG (1911) The intrinsic factors in the act of progression in the mammal. Proc R Soc Lond B Biol Sci 84:308-319.

Calancie B, Needham-Shropshire B, Jacobs P, Willer K, Zych G, Green BA (1994) Involuntary stepping after chronic spinal cord injury. Evidence for a central rhythm generator for locomotion in man. Brain 117:1143-1159.

Chambers WW, Sprague JM (1955a) Functional localization in the cerebellum I. Organization in longitudinal cortico-nuclear zones and their contribution to the control of posture, both extrapyramidal and pyramidal. J Comp Neurol 103:105-130.

Chambers WW, Sprague JM (1955b) Functional localization in the cerebellum II. Somatotopic organization in cortex and nuclei. Arch Neurol Psychiatry 74:653-680.

Dichgans J, Diener HC (1985) Clinical evidence for functional compartmentalization of the cerebellum. In: Cerebellar functions (Bloedel JR, Dichgans J, Precht W, eds), pp 126-147. Berlin: Springer.

Dietz V, Zijlstra W, Duysens J (1994) Human neuronal interlimb coordination during split-belt locomotion. Exp Brain Res 101:513-520.

Dimitrijevic MR, Gerasimenko Y, Pinter MM (1998) Evidence for a spinal central pattern generator in humans. Ann NY Acad Sci 860:360-376.

Duysens J, Pearson KG (1980) Inhibition of flexor burst generation by loading ankle extensor muscles in walking cats. Brain Res 187:321-332.

Earhart GM, Bastian AJ (2001) Selection and coordination of human locomotor forms following cerebellar damage. J Neurophysiol 85:759-769.

Eccles JC, Oscarsson O, Willis WD (1961) Synaptic action of group I and II afferent fibres of muscles on cells of the dorsal spinocerebellar tract. J Physiol (Lond) 158:517-543.

Forssberg H, Grillner S, Rossignol S (1975) Phase dependent reflex reversal during walking in chronic spinal cats. Brain Res 85:103-107.

Forssberg H, Grillner S, Halbertsma, Rossignol S (1980) The locomotion of the low spinal cat. II. Interlimb coordination. Acta Physiol Scand 108:283-295.

Grillner S (1985) Neurobiological bases of rhythmic motor acts in vertebrates. Science 228:143-149.

Grillner S, Rossignol S (1978) On the initiation of the swing phase of locomotion in chronic spinal cats. Brain Res 146:269-277.

Grillner S, Wallen P (1985) Central pattern generators for locomotion, with special reference to vertebrates. Annu Rev Neurosci 8:233-2361.

Grillner S, Zangger P (1979) On the central generation of locomotion in the low spinal cat. Exp Brain Res 34:241-261.

Grillner S, Deliagina T, Ekeberg O, el Manira A, Hill RH, Lansner A, Orlovsky GN (1995) Neural networks that co-ordinate locomotion and body orientation in lamprey. Trends Neurosci 18:270-279.

Hansel C, Linden DJ, D’Angelo E (2001) Beyond parallel fiber LTD: the diversity of synaptic and non-synaptic plasticity in the cerebellum. Nat Neurosci 4:467-475.

Horak FB, Diener HC (1994) Cerebellar control of postural scaling and central set in stance. J Neurophysiol 72:479-493.

Imamizu H, Miyauchi S, Tamada T, Sasaki Y, Takino R, Putz B, Yoshioka T, Kawato M (2000) Human cerebellar activity reflecting an acquired internal model of a new tool. Nature 403:192-195.

Ito M (1989) Long-term depression. Annu Rev Neurosci 12:85-102.

Ito M (2000) Mechanisms of motor learning in the cerebellum. Brain Res 886:237-245

Kawato M, Gomi H (1992) A computational model of four regions of the cerebellum based on feedback-error learning. Biol Cybern 68:95-103.

Kulagin AS, Shik ML (1970) Interaction of symmetric extremities during controlled locomotion (in Russian). Biofizika 15:164-170.

Lam T, Anderschitz M, Dietz V (2006) Contribution of feedback and feedforward strategies to locomotor adaptations. J Neurophysiol 95:766-773. 
Lang CE, Bastian AJ (1999) Cerebellar subjects show impaired adaptation of anticipatory EMG during catching. J Neurophysiol 82:2108-2119.

Lennard PR, Stein PS (1977) Swimming movements elicited by electrical stimulation of turtle spinal cord. I. Low-spinal and intact preparations. J Neurophysiol 40:768-778.

Lou JS, Bloedel JR (1992) Responses of sagittally aligned Purkinje cells during perturbed locomotion: synchronous activation of climbing fiber inputs. J Neurophysiol 68:570-580.

Lundberg A, Oscarsson O (1956) Functional organization of the dorsal spino-cerebellar tract in the cat. IV. Synaptic connections of afferents from Golgi tendon organs and muscle spindles. Acta Physiol Scand 38:53-75.

Lundberg A, Winsbury G (1960) Functional organization of the dorsal spinocerebellar tract. VI. Further experiments on excitation from tendon organ and muscle spindle afferents. Acta Physiol Scand 49:165-170.

Martin TA, Keating JG, Goodkin HP, Bastian AJ, Thach WT (1996) Throwing while looking through prisms II. Specificity and storage of multiple gaze-throw calibrations. Brain 119:1199-1211.

Maschke M, Gomez CM, Ebner TJ, Konczak J (2004) Hereditary cerebellar ataxia progressively impairs force adaptation during goal-directed arm movements. J Neurophysiol 91:230-238.

Matsukawa K, Kamei H, Minoda K, Udo M (1982) Interlimb coordination in cat locomotion investigated with perturbation. I. Behavioral and electromyographic study on symmetric limbs of decerebrate and awake walking cats. Exp Brain Res 46:425-437.

McCormick DA, Steinmetz JE, Thompson RF (1985) Lesions of the inferior olivary complex cause extinction of the classically conditioned eyeblink response. Brain Res 359:120-130.

Morton SM, Bastian AJ (2003) Relative contributions of balance and voluntary leg-coordination deficits to cerebellar gait ataxia. J Neurophysiol 89:1844-1856.

Morton SM, Bastian AJ (2004) Cerebellar adaptation of locomotion: impaired adaptation during split-belt but not coupled treadmill locomotion. Soc Neurosci Abstr 30:415.13.

Nowak DA, Hermsdorfer J, Rost K, Timmann D, Topka H (2004) Predictive and reactive finger force control during catching in cerebellar degeneration. Cerebellum 3:227-235.

Pang MY, Yang JF (2000) The initiation of the swing phase in human infant stepping: importance of hip position and leg loading. J Physiol (Lond) 528:389-404.

Pearson KG (1995) Proprioceptive regulation of locomotion. Curr Opin Neurobiol 5:786-791.

Pearson KG (2000) Neural adaptation in the generation of rhythmic behavior. Annu Rev Physiol 62:723-753.

Poppele RE, Rankin A, Eian J (2003) Dorsal spinocerebellar tract neurons respond to contralateral limb stepping. Exp Brain Res 149:361-370.

Reisman DS, Block HJ, Bastian AJ (2005) Interlimb coordination during locomotion: what can be adapted and stored? J Neurophysiol 94:2403-2415.

Roberts A, Tunstall MJ, Wolf E (1995) Properties of networks controlling locomotion and significance of voltage dependency of NMDA channels: stimulation study of rhythm generation sustained by positive feedback. J Neurophysiol 73:485-495.

Rossignol S, Chau C, Brustein E, Giroux N, Bouyer L, Barbeau H, Reader TA (1998) Pharmacological activation and modulation of the central pattern generator for locomotion in the cat. Ann NY Acad Sci 860:346-359.

Rossignol S, Drew T, Brustein E, Jiang W (1999) Locomotor performance and adaptation after partial or complete spinal cord lesions in the cat. Prog Brain Res 123:349-365.

Shadmehr R, Mussa-Ivaldi FA (1994) Adaptive representation of dynamics during learning of a motor task. J Neurosci 14:3208-32024.

Shimansky Y, Wang J-J, Bauer RA, Bracha V, Bloedel JR (2004) On-line compensation for perturbations of a reaching movement is cerebellar dependent: support for the task dependency hypothesis. Exp Brain Res 155:156-172.

Smith MA, Shadmehr R (2005) Intact ability to learn internal models of arm dynamics in Huntington's disease but not cerebellar degeneration. J Neurophysiol 93:2809-2821.

Smith MA, Brandt J, Shadmehr R (2000) Motor disorder in Huntington's disease begins as a dysfunction in error feedback control. Nature 403:544-549.

Sprague JM, Chambers WW (1953) Regulation of posture in intact and decerebrate cat. I. Cerebellum, reticular formation, vestibular nuclei. J Neurophysiol 16:451-463.

Thach WT, Goodkin HP, Keating JG (1992) The cerebellum and the adaptive coordination of movement. Annu Rev Neurosci 15:403-442.

Thelen E, Ulrich BD, Niles D (1987) Bilateral coordination in human infants: stepping on a split-belt treadmill. J Exp Psychol Hum Percept Perform 13:405-410.

Trouillas P, Takayanagi T, Hallett M, Currier RD, Subramony SH, Wessel K, Bryer A, Diener HC, Massaquoi S, Gomez CM, Coutinho P, Ben Hamida M, Campanella G, Filla A, Schut L, Timann D, Honnorat J, Nighoghossian N, Manyam B (1997) International cooperative ataxia rating scale for pharmacological assessment of the cerebellar syndrome. J Neurol Sci 145:205-211.

Weiner MJ, Hallett M, Funkenstein HH (1983) Adaptation to lateral displacement of vision in patients with lesions of the central nervous system. Neurol 33:766-772.

Yanagihara D, Kondo I (1996) Nitric oxide plays a key role in adaptive control of locomotion in cat. Proc Natl Acad Sci USA 93:13292-13297.

Yanagihara D, Udo M (1994) Climbing fiber responses in cerebellar vermal Purkinje cells during perturbed locomotion in decerebrate cats. Neurosci Res 19:245-248.

Yanagihara D, Udo M, Kondo I, Yoshida T (1993) A new learning paradigm: adaptive changes in interlimb coordination during perturbed locomotion in decerebrate cats. Neurosci Res 18:241-244.

Yang JF, Stephens MJ, Vishram R (1998) Transient disturbances to one limb produce coordinated, bilateral responses during infant stepping. J Neurophysiol 79:2329-2337.

Yang JF, Lam T, Pang MY, Lamont E, Musselman K, Seinen E (2004) Infant stepping: a window to the behaviour of the human pattern generator for walking. Can J Physiol Pharmacol 82:662-674. 